\title{
Circulating Inflammation Markers Partly Explain the Link Between the Dietary Inflammatory Index and Depressive Symptoms
}

\author{
Alessandro Gialluisi $\mathbb{D}^{1}$ \\ Federica Santonastaso (iD) 2 \\ Marialaura Bonaccio' \\ Francesca Bracone \\ Nitin Shivappa ${ }^{3,4}$ \\ James R Hebert ${ }^{3,4}$ \\ Chiara Cerletti ${ }^{\prime}$ \\ Maria Benedetta Donati (D) \\ Giovanni de Gaetano' \\ Licia lacoviello ${ }^{1,2}$ \\ On behalf of the Moli-sani \\ Investigators

\begin{abstract}
'Department of Epidemiology and Prevention, IRCCS Neuromed, Pozzilli, Italy; ${ }^{2}$ Department of Medicine and Surgery, University of Insubria, Varese, Italy; ${ }^{3}$ Cancer Prevention and Control Program and Department of Epidemiology and Biostatistics, Arnold School of Public Health, University of South Carolina, Columbia, SC, USA; ${ }^{4}$ Department of Nutrition, Connecting Health Innovations LLC, Columbia, SC, USA
\end{abstract}

Background: Depression is a mood disorder characterized by a high rate of resistance to pharmacological treatments, which has often been linked to chronic inflammation. This can be influenced by different environmental factors, in particular pro-inflammatory diets. However, a mediating role of circulating inflammation has never been observed.

Aim: To test the association between a dietary inflammatory index (DII ${ }^{\mathbb{B}}$ ) and continuous depressive symptoms (adapted version of PHQ9) in an Italian population cohort $(\mathrm{N}=13,301)$, along with potential explanatory effect of a composite index (INFLA-score) based on four circulating inflammatory biomarkers: C-reactive protein, granulocyte-to-lymphocyte ratio, platelet and white blood cell counts.

Results: Significant positive associations were observed between DII and total depressive symptoms (standardized $\beta(\mathrm{SE})=0.038(0.005), \mathrm{p}<0.001)$, and with two factors tagging somatic $(0.012(0.003), \mathrm{p}<0.001)$ and cognitive symptoms $(0.012(0.003), \mathrm{p}<0.001)$, after adjustment for different potential confounders (socioeconomic status, chronic health conditions and lifestyles). These associations were about twice as strong in women than in men. INFLA-score explained a small but significant proportion of the association with total depressive symptoms $(0.90-2.30 \%, \mathrm{p}<0.05)$, which was mainly driven by granulocyte-tolymphocyte ratio $(1.18-1.65 \%)$. This effect was even stronger for the somatic $(2.66-4.66 \%)$ but not for the cognitive factor $(0 \%)$.

Conclusion: These findings support a strong link between inflammatory diet and depression, especially with somatic symptoms and within women. Moreover, they provide novel evidence for a potential explanatory role of circulating inflammation in this association, suggesting new paths for prevention and treatment of major and atypical depression.

Keywords: dietary inflammatory index, depressive symptoms, atypical depression, circulating inflammation, granulocyte-to-lymphocyte ratio, white blood cells

\section{Introduction}

Depression is one of the most disabling chronic disorders, affecting over 300 million people worldwide, ${ }^{1}$ with a lifelong prevalence of about $20 \%$ in the United States. $^{2}$ While the etiology of this disease has been classically associated with the disruption of several neurotransmitters like serotonin, dopamine and glutamate, ${ }^{3}$ conventional treatments targeting these neurotransmitter pathways such as selective serotonin reuptake inhibitors - are effective only in one-third of the cases, with frequent relapsing episodes. ${ }^{4}$ This applies especially to "atypical" depression, which is characterized by somatic/neurovegetative symptoms like
Correspondence: Alessandro Gialluisi Department of Epidemiology and Prevention, IRCCS Neuromed, Via dell 'Elettronica, 86077, Pozzilli, Italy Tel +390865915244

Email alessandro.gialluisi@gmail.com 
fatigue, psychomotor retardation, altered sleep and appetite. $^{5-7}$ This subtype of depression has often and robustly been linked to systemic inflammation by independent lines of evidence, ${ }^{7-10}$ underlining the importance of healthy lifestyles to reduce the risk of this disease and to possibly treat it. ${ }^{11}$ In particular, dietary habits and nutritional patterns have shown a prominent influence on the risk of depression, ${ }^{12,13}$ as well as on circulating inflammation. $^{14-16}$ For this reason, dietary interventions promoting the intake of anti-inflammatory compounds has been suggested to prevent depression. ${ }^{13}$

A handful of studies have specifically tested the association between the inflammatory potential of diet - often assessed through Dietary Inflammatory Index $\left(\mathrm{DII}^{\circledR}\right)^{17}$ and depression risk, quite consistently revealing a direct association. $^{12,13}$ Most of these studies also reported significant associations of pro-inflammatory diets and circulating levels of a number of inflammation markers such as C-reactive protein (CRP), interleukin-6 (IL-6) and other cytokines like Tumor Necrosis Factor $\alpha$ (TNF- $\alpha),{ }^{18-20}$ although results are inconsistent. ${ }^{21}$ Only a few studies have tested a potential mediating role of circulating inflammation in this link (reviewed in ${ }^{17}$ ). Akbaraly et al ${ }^{18}$ reported a positive association of high DII scores (indicating a pro-inflammatory diet) with higher circulating IL-6 and CRP levels, as well as with a higher risk of recurrent depressive symptoms, in middle-aged women of the Whitehall II study (35-55 years). However, this latter association was not significantly explained by the former. ${ }^{18}$ Phillips et al analyzed a broader outcome, mental health, assessing depression, anxiety and wellbeing scales, in an Irish cohort (50-69 years), again finding no evidence of changes in the magnitude of associations when further adjusting for CRP, TNF-a and IL6 concentrations. ${ }^{19}$ Jorgensen et $\mathrm{al}^{20}$ observed that depressed subjects from the NHANES 2007-2012 cohort (age > 18 years) had higher CRP concentrations, which was in turn positively associated with dietary inflammation. Although they reported a significant explanatory effect for a cardiovascular risk score in the link between depression and DII, they did not provide evidence for a formal mediation of CRP. ${ }^{20}$ Vermeulen et al reported Italian elders $(\geq$ 65 years) in the highest quartile of a specific inflammatory dietary pattern to have higher TNF- $\alpha$ and CRP circulating levels; however, they concluded that such a link was not explained by the association between this dietary pattern and circulating inflammatory markers. ${ }^{21}$
Because no study so far reported robust evidence supporting a role of circulating inflammation in the link between dietary inflammation and depression, ${ }^{17}$ this study was designed to test this hypothesis within a large Italian population cohort, the Moli-sani study. ${ }^{22}$ To this end, we first tested cross-sectional association between the inflammatory content of diet and a continuous score of depressive symptoms, and then quantified potential explanatory effects of a composite index of circulating inflammation - the INFLA-score - based on cytokinerelated, hemostatic and cellular components of inflammation. ${ }^{23}$ Finally, we repeated the same analysis with two factors tagging cognitive and somatic depressive symptoms, so to possibly detect domain-specific associations. This study represents an advance compared to our previous analysis of symptoms-specific associations with circulating inflammation in the Moli-sani study, ${ }^{7}$ since here we add a further piece to the puzzle, namely the relation between dietary inflammation and DII, and then investigate the role of circulating inflammation in this link, using both an overall scale of depressive symptoms and a factor-based approach. Moreover, we investigate associations stratified by gender and chronic health conditions.

\section{Materials and Methods Population of Study}

The study population consisted of adult subjects participating to the Moli-sani project ( $\mathrm{N}=24,325 ; 48.11 \%$ men), a cohort of Italian residents who were randomly recruited from the general population of Molise Region (CentralSouthern Italy) in two centers (Termoli and Campobasso), between March 2005 and April 2010. Exclusion criteria were pregnancy at the time of recruitment, inability to understand terms of participation, current poly-traumas (ie, simultaneous injury to several organs or body systems), coma, or refusal to sign the informed consent. ${ }^{22}$ The Moli-sani Study was approved by the ethical committee of the Catholic University of Rome (approval nr: A-931/03-138-04/CE 2004) and all the participants provided written informed consent. For this study, no formal approval was required by the steering committee since an internal researcher was leading the analyses and all the members of the committee were contributing to the study. Further details on the implementation procedure of the whole project are reported on the Moli-sani website (see URLs). 
Participants with complete questionnaire data on depression symptoms were retrieved $(\mathrm{N}=13,776)$. Then, subjects with unreliable medical or dietary questionnaires were removed $(\mathrm{n}=355)$, as well as individuals with missing data for at least one of the low-grade inflammation biomarkers tested $(\mathrm{n}=58)$ and those with unreliable blood marker levels (sum of leukocyte fraction $<99 \%$ or $>101 \%, n=49$ ). Lastly, participants with missing information in at least one of basic socio-demographic covariates (age, sex and education) were filtered out $(n=13)$. This resulted in a final analyzed sample size of 13,301 subjects with evaluable data. ${ }^{7}$

\section{Psychometric Assessment}

To assess depressive symptoms, the Patient Health Questionnaire 9 (PHQ9) was administered to a subset of the Moli-sani participants, as in. ${ }^{7,11,24}$ The PHQ9 scale assesses the severity of depression by scoring the frequency of nine specific domains, typically affecting depressed subjects, in the two weeks before the interview. These include anhedonia, low mood, alteration of sleeping patterns or eating behaviors, feeling of failure/low self-estimate, fatigue, troubles in mental concentration, hypo-/hyperactivity behaviors, and suicidal ideation. ${ }^{25}$ However, we used a reduced version of PHQ9 with 8 items (hereafter called PHQ9-6), because of unavailable information about feeling of failure. This score, ranging from 0 (indicating no depressive symptoms at all) to 24 (suggestive of severe depression), was validated for the screening of different depression severity forms in the Moli-sani study, showing a performance comparable with the gold-standard (PHQ9). ${ }^{24,26}$

\section{Inflammatory Markers}

We tested circulating inflammation as a variable possibly explaining the association between dietary inflammation and depressive symptoms. Specifically, we used a composite blood-based inflammation index, called INFLA-score, based on four circulating biomarkers C-reactive protein levels (CRP), blood platelet count (Plt), white blood cell count (WBC), and granulocyte-tolymphocyte ratio (GLR) - and capturing both serum and cellular-circulating inflammation. ${ }^{23}$ This score has been already validated as a comprehensive index of circulating inflammation, since it includes cytokine-related, hemostatic and immune components of the inflammatory response, ${ }^{11}$ and has been previously associated with the inflammatory potential of diet (assessed as below) within the Moli-sani study. ${ }^{14}$ Of note, blood samples were collected after overnight fasting, which reduced the potential inflammatory influence of meals consumed immediately prior to the blood draw.

\section{Dietary Inflammatory Index (DII $\left.{ }^{\circledR}\right)$}

DII is a dietary score indicating the pro- and antiinflammatory potential of the overall diet. ${ }^{17}$ This was designed and validated to evaluate whole-diet inflammatory capacity, according to the pro- and anti-inflammatory efficacy of different dietary components on various circulating inflammatory biomarkers. ${ }^{18}$ More specifically, this tool scores diets based on up to 45 food components and compares individual self-reports to comparative data from 11 food consumption data sets from Countries around the world. This produces a more appropriate tool for determining dietary inflammatory potential, ${ }^{17}$ with a higher DII score indicating greater proinflammatory potential of the diet.

For the present study, dietary information derived from the Italian EPIC Food Frequency Questionnaire was used to calculate scores for all subjects, as described in. ${ }^{14}$ Briefly, dietary data for each participant were first linked to a regionally representative global database, which provides a robust estimate for each dietary intake analyzed (mean and standard deviation). A z-score was derived by subtracting the "standard global mean" from the amount reported and then dividing this value by the standard deviation. To minimize the effect of "right skewing", this value was converted to a centered proportion, which was then multiplied by the respective food parameter inflammatory effect score (derived from a literature review and scoring of 1943 "qualified" articles), to obtain the subject's food parameter-specific score. All resulting scores were then summed up to create the overall DII score for every subject in the study. For the current study, data were available for a total of 33 food parameters, including carbohydrate, protein, total fat, alcohol, fiber, cholesterol, saturated fat, monounsaturated fat, polyunsaturated fat, omega-3, omega-6 fatty acid, niacin, thiamin, riboflavin, vitamin $\mathrm{B} 12$, vitamin $\mathrm{B} 6$, iron, magnesium, zinc, vitamin $A$, vitamin $C$, vitamin $D$, vitamin $E$, folic acid, $\beta$-carotene, anthocyanidins, flavanol, flavones, flavonols, flavonones, isoflavones, garlic/onion and tea. A classification of these parameters in pro- and anti-inflammatory foods is reported in Table S1.

\section{Statistical Analyses}

Statistical analyses were carried out $\mathrm{inR}^{27}$ to test the association between DII (dietary exposure) and 
standardized PHQ9-6 (psychometric outcome, divided by its standard deviation), through linear regression models $(\operatorname{lm}()$ function). While all models were adjusted for recruiting center, two main incremental models were built, one adjusting for age and sex (Model 1) and one further adjusting for additional variables which may act as a confounders in the relationship between systemic inflammation and depression, including socioeconomic status (proxied by completed education level), lifestyles (drinking and smoking habits, leisure-time physical activity) and prevalent chronic conditions (cancer, diabetes and cardiovascular disease). ${ }^{28-32}$ Sensitivity models were built further adjusting for psychological resilience assessed through the Connor-Davidson Resilience Scale (CDRISC; ${ }^{33}$ Model 3); adherence to Mediterranean Diet $^{34}$ and daily caloric intake (Model 4); adiposity assessed through Relative Fat Mass ${ }^{35}$ (Model 5) and self-reported use of antidepressants (Model 6). This was done with the aim of partialling out further potential confounding (eg, antidepressants, resilience, Mediterranean diet and caloric intake) or overlapping mediating effects on the observed associations (eg, resilience, fat mass). Indeed, in the Molisani study adherence to Mediterranean Diet was positively associated with psychological resilience, ${ }^{36}$ defined as the ability to cope with stress, which acts as a sort of buffering capacity to lower depression and anxiety under stressful situations. While a confounder effect of Mediterranean Diet or use of antidepressants can be hypothesized in the association between DII and depressive symptoms, it is unclear whether resilience plays a confounder or a mediation role in this relationship. Still, here we aimed to identify specific associations of DII with psychological domains strictly related to depressive symptoms, which prompted us to perform sensitivity analyses adjusting for resilience. Similarly, adiposity may play a mediator role since it can be clearly influenced by fat-rich (proinflammatory) diets and adipose tissues contribute to the secretion of inflammatory cytokines, ${ }^{37}$ hence we included RFM adjustment in sensitivity models to detect potential fat-independent associations. A detailed description of these variables is reported in Supplementary Methods. Association analyses were also performed stratifying the available samples by gender, use of antidepressants and prevalent health conditions (CVD, cancer, diabetes and multimorbidity), classified into binary categories (Yes/No).

Finally, through the mediate() function of the mediation package (see URLs), we estimated the proportion of total effect (PTE) of dietary inflammation on depressive symptoms which was explained by the INFLA-score and by each single component biomarker. To define the exact percentage of the association which could be specifically ascribed to inflammation, we performed two distinct analyses for each biomarker tested: a basic model adjusted for age and sex (Model 1), and one that further adjusted for education, lifestyles and health conditions (Model 2).

\section{Factor Analysis of Depressive Symptoms}

To determine whether dietary inflammation associated more strongly with specific domains of depression - representing cognitive and neurovegetative symptoms - we carried out a polychoric factor analysis of all depressive symptoms assessed in the population of study, through the psych package (see URLs). Specifically, we first computed a polychoric correlation matrix (Figure S1), then we applied the $\mathrm{fa}()$ function to compute two factors (applying oblimin rotation) and analyzed the loadings of each depressive domain assessed (see Results below). Each of the two factors resulting from the analysis was then analyzed as above, first testing linear associations with DII and then estimating the proportion of total effect explained by INFLA-score and its component biomarkers in these associations. For each of the factors tested, all models were adjusted as above, and for the other factor not used as an outcome to detect associations which were not driven by the shared variance between the depressive domains they represented. Similarly, PTEs and stratified associations were tested as for overall depressive symptoms.

\section{Results}

The characteristics of the population under study are reported in Table 1 . The analyzed subjects were more prevalently men $(49.3 \%$ vs $46.7 \% ; p<0.001)$, younger (mean (SD) age: 53.2 (10.9) vs 59.0 (12.4) years; $p<$ $0.001)$ and more educated $(p<0.001)$, compared to those removed from the analysis. Prevalent health conditions were less frequent in the analyzed subset $(p<0.001)$, which showed also a less frequent use of drugs with potential anti-inflammatory action (eg, antihyperlipidemic, antihypertensive and anticoagulant drugs, $p<0.001$ ). However, no significant difference in the use of antidepressants or in the self-reported history of depression or other psychiatric disorder was observed. Similarly, there was no difference in the average INFLA-score and WBC, but a lower CRP, a higher Plt and GLR in the analyzed subset $(p<0.001$; Table 1$)$. 
Table I Characteristics of the Population Under Study (After QC, N=|3,30I) vs Participants Removed from the Analysis (N=II,024) and vs the Whole Moli-Sani Cohort $(\mathrm{N}=24,325)$

\begin{tabular}{|c|c|c|c|c|}
\hline Variable & $\begin{array}{c}\text { Population Under } \\
\text { Study }\end{array}$ & $\begin{array}{c}\text { Removed } \\
\text { Participants }\end{array}$ & $\begin{array}{l}\text { Moli-Sani } \\
\text { Cohort }\end{array}$ & $\begin{array}{c}\text { P for } \\
\text { Difference }\end{array}$ \\
\hline $\mathrm{N}$ & $|3,30|$ & $1 \mathrm{I}, 024$ & 24,325 & - \\
\hline Sex (males) & $49.3 \%$ & $46.7 \%$ & $48.1 \%$ & $* * *, *$ \\
\hline Age $(y ;$ mean, sd) & $53.2(10.9)$ & $59.0(12.4)$ & $55.8(12.0)$ & $* * *, * * *$ \\
\hline $\begin{array}{l}\text { Education } \\
\text { Primary } \\
\text { Lower secondary } \\
\text { Upper secondary } \\
\text { Post-secondary } \\
\text { Unknown }\end{array}$ & $\begin{array}{c}15.1 \% \\
29.7 \% \\
40.2 \% \\
14.9 \% \\
\text { Removed }\end{array}$ & $\begin{array}{c}38.6 \% \\
25.3 \% \\
26.4 \% \\
9.3 \% \\
0.4 \%\end{array}$ & $\begin{array}{c}25.8 \% \\
27.7 \% \\
34.0 \% \\
12.4 \% \\
0.2 \%\end{array}$ & $* * *, * * *$ \\
\hline $\begin{array}{l}\text { Health conditions } \\
\text { CVD } \\
\text { Cancer } \\
\text { Diabetes } \\
\text { Self-reported depression or other psychiatric } \\
\text { disorder }\end{array}$ & $\begin{array}{c}4.5 \% \\
2.9 \% \\
3.9 \% \\
11.5 \%\end{array}$ & $\begin{array}{c}7.5 \% \\
3.6 \% \\
6.3 \% \\
11.8 \%\end{array}$ & $\begin{array}{l}5.9 \% \\
3.2 \% \\
5.0 \% \\
11.6 \%\end{array}$ & $\begin{array}{l}* * *, * * * \\
* * *, * * * \\
* * *, * * * \\
\text { ns, ns }\end{array}$ \\
\hline $\begin{array}{l}\text { Lifestyle factors } \\
\text { Current Smokers } \\
\text { Regular Drinkers } \\
\text { Energy intake (Kcal/d; mean, sd) } \\
\text { MeDi score (mean, sd) } \\
\text { Physical activity (meth/d; mean, sd) }\end{array}$ & $\begin{array}{c}24.2 \% \\
62.5 \% \\
2139(631) \\
4.4(1.7) \\
3.4(3.8)\end{array}$ & $\begin{array}{c}21.5 \% \\
57.4 \% \\
2006(703) \\
4.3(1.6) \\
3.6(4.3)\end{array}$ & $\begin{array}{c}27.4 \% \\
60.2 \% \\
2079(668) \\
4.3(1.6) \\
3.5(4.0)\end{array}$ & $\begin{array}{c}* * *, * * * \\
* * *, * * * \\
* * *, * * * \\
\mathrm{~ns}, \mathrm{~ns} \\
* * *, * *\end{array}$ \\
\hline Obesity (RFM; mean, sd) & $34.80(7.59)$ & $36.34(8.01)$ & $35.50(7.82)$ & $* * *, * * *$ \\
\hline $\begin{array}{l}\text { Inflammation (mean, sd) } \\
\text { INFLA-score } \\
\text { CRP (mg/L) } \\
\text { PIt }\left(\times 10^{9} \text { platelets } / \mathrm{L}\right) \\
\text { WBC }\left(\times 10^{9} \text { leukocytes/L) }\right. \\
\text { GLR }\end{array}$ & $\begin{array}{c}-0.12(5.96) \\
2.44(3.10) \\
252.07(63.31) \\
6.23(1.73) \\
1.96(0.78)\end{array}$ & $\begin{array}{c}-0.05(6.12) \\
2.78(3.44) \\
244.86(64.99) \\
6.23(1.84) \\
2.04(1.08)\end{array}$ & $\begin{array}{c}-0.09(6.03) \\
2.59(3.26) \\
248.92(64.15) \\
6.23(1.78) \\
2.02(0.93)\end{array}$ & $\begin{array}{c}\mathrm{ns}, \mathrm{ns} \\
* * *, * * * \\
* * *, * * * \\
\mathrm{~ns}, \mathrm{~ns} \\
* * *, *\end{array}$ \\
\hline $\begin{array}{l}\text { Anti-inflammatory medications use } \\
\text { Antidepressant } \\
\text { Antihyperlipidemic } \\
\text { Antihypertensive } \\
\text { Anticoagulant } \\
\text { Respiratory anti inflammatory }\end{array}$ & $\begin{array}{c}3.0 \% \\
6.5 \% \\
24.0 \% \\
4.7 \% \\
0.3 \%\end{array}$ & $\begin{array}{c}3.0 \% \\
9.4 \% \\
34.4 \% \\
8.5 \% \\
0.6 \%\end{array}$ & $\begin{array}{c}3.0 \% \\
7.9 \% \\
28.7 \% \\
6.4 \% \\
0.4 \%\end{array}$ & $\begin{array}{l}\mathrm{ns}, \mathrm{ns} \\
* * *, * * * \\
* * *, * * * \\
* * *, * * * \\
* *, \mathrm{~ns}\end{array}$ \\
\hline
\end{tabular}

Notes: P-values resulting from statistical comparisons of the analysed vs non-analysed participants and vs the whole Moli-sani cohort are reported $(* * * \mathrm{p}<0.00 \mathrm{I} ; * * \mathrm{p}<0.0 \mathrm{I}$; ${ }^{*} \mathrm{p}<0.05$; ns = non-significant). Chi-squared test was applied to education levels, CVD, cancer, smoking and drinking classes, Fisher Exact Test to sex, diabetes, and use of anti-inflammatory medications, unpaired $t$-test to age, INFLA-score, PIt, GLR, MeDi score and caloric intake, and Wilcoxon signed rank test to CRP, WBC, RFM and physical activity levels (see Supplementary Methods for further details on the definition of these covariates).

Abbreviations: $C \overline{V D}$, cardiovascular disease; MeDi, adherence score to Mediterranean Diet (MeDi); ${ }^{34}$ CRP, C-reactive protein; Plt, platelets count; WBC, white blood cells count; GLR, granulocyte-to-lymphocyte ratio; RFM, Relative Fat Mass. ${ }^{35 .}$

In this cohort, we observed a significant association between PHQ9-6 and the DII (Table 2), both in the basic model (Model 1; $\beta(\mathrm{SE})=0.043(0.005), p<0.01)$ and in a model further adjusted for socioeconomic status, lifestyles and chronic health conditions (Model 2; $\beta$ (SE) $=0.038$ (0.005), $p<0.01)$. The observed association survived correction for psychological resilience (Model $3 ; \beta(\mathrm{SE})=0.027(0.006), p<0.01)$, adherence to 
Table 2 Associations Between Dietary Inflammatory Index (DII) and Depressive Symptoms in the Analyzed Cohort (N=I3,30I)

\begin{tabular}{|c|c|c|c|c|}
\hline Model & Covariates & $\begin{array}{l}\text { Overall Symptoms (PHQ9- } \\
\text { 6) } \beta \text { (SE), p-value }\end{array}$ & $\begin{array}{l}\text { Somatic Factor } \beta \\
\text { (SE), p-value }\end{array}$ & $\begin{array}{l}\text { Cognitive Factor } \beta \\
\text { (SE), p-value }\end{array}$ \\
\hline 1 & Age + sex & $0.043(0.005),<2.0 \times 10^{-16}$ & $\begin{array}{c}0.013(0.003), 3.2 \times \\
10^{-4}\end{array}$ & $0.011(0.003), 0.003$ \\
\hline 2 & $\begin{array}{c}\text { Model I + education }+ \text { PA + smoking + drinking + } \\
\text { diabetes }+ \text { cancer + CVD }\end{array}$ & $0.038(0.005), 2.0 \times 10^{-14}$ & $\begin{array}{c}0.012(0.003), 8.8 \times \\
10^{-4}\end{array}$ & $0.009(0.004), 0.01$ \\
\hline 3 & Model $2+$ resilience & $0.027(0.006), 1.6 \times 10^{-6}$ & $\begin{array}{l}0.011(0.004) \\
0.009\end{array}$ & $0.004(0.004), 0.29$ \\
\hline 4 & Model $2+M e D i+$ daily energy intake & $0.031(0.006), 3.0 \times 10^{-7}$ & $0.010(0.043), 0.02$ & 0.007 (0.004), 0.13 \\
\hline 5 & Model $2+$ RFM & $0.039(0.005), 4.2 \times 10^{-15}$ & $\begin{array}{c}0.012(0.003), 7.3 \times \\
10^{-5}\end{array}$ & $0.004(0.001), 0.006$ \\
\hline 6 & Model $2+$ antidepressants & $0.037(0.005), 4.2 \times 10^{-14}$ & $\begin{array}{c}0.036(0.005), 1.3 \times \\
10^{-12}\end{array}$ & $\begin{array}{c}0.035(0.005), 6.8 \times \\
10^{-12}\end{array}$ \\
\hline
\end{tabular}

Notes: All models were adjusted for recruiting center, in addition to the above mentioned covariates (see Supplementary Methods for detailed definitions). When factors were modelled as outcome, the factor other than the outcome was also included among covariates. Beta coefficients and Standard Errors (SE) are reported for standardized PHQ9-6 and factor scores associated to unitary increase of DIl, along with relevant $p$-values. Significant associations $(p<0.05)$ are highlighted in bold.

Abbreviations: PA, leisure-time physical activity; CVD, cardiovascular disease; MeDi, adherence score to Mediterranean Diet; ${ }^{34}$ RFM, Relative Fat Mass. $^{35 .}$

Mediterranean Diet and caloric intake (Model 4; $\beta$ (SE) $=$ 0.031 (0.006), $p<0.01$ ), relative fat mass (Model $5 ; \beta$ (SE) $=0.039(0.005), p<0.01)$ and use of antidepressants (Model 6; $\beta$ (SE) $=0.037$ (0.005), $p<0.01$ ). Genderstratified analysis revealed a consistently stronger association between DII and depressive symptoms in women compared to men, both in raw models (see Figure 1A and $\mathrm{B}$ ) and in models adjusted for age, lifestyles and health conditions (Table S2A). Similarly, analyses stratified by health conditions and use of antidepressants showed an almost null association for subjects with prevalent CVD and multimorbidity, while those affected by cancer and diabetes and antidepressant users showed associations consistent with the whole population under study (Figure 1C and Table S2B).

A small but significant proportion of the association between PHQ9-6 and DII was explained by INFLAscore (Table 3), both in a basic (PTE $=2.30 \% ; p<$ 0.01 ) and in a full model adjusted for socioeconomic, lifestyle and health conditions ( $\mathrm{PTE}=0.90 \% ; p=0.04$ ). Among single component biomarkers of INFLA-score, WBC and GLR showed the most significant contribution to this effect, explaining $0.99 \%$ and $1.65 \%$ of the association in the basic model $(p \leq 0.01)$, while CRP and Plt did not reveal significant explanatory effects. In the full model, proportions of association explained by WBC and GLR were milder $-0.008 \%$ and $1.18 \%$, respectively
- and still significant only for GLR ( $p=0.01$; see Table 3).

A polychoric factor analysis of single PHQ9-6 items revealed two main factors, explaining $35.9 \%$ and $17.1 \%$ of the variance shared among all depressive symptoms (Figure S2), tagging neurovegetative/somatic and cognitive/affective domains, respectively (see loadings in Figure S3). When we modelled each of these factors as a function of DII (reciprocally adjusting for the other factor), the "somatic" factor showed a stably significant association (Model 1: $\beta$ (SE) $=0.013$ (0.003), $p<0.01$; Model 2: $\beta(\mathrm{SE})=0.012(0.003), p<0.01)$, which held across all tested models (Table 2). INFLA-score explained a significant proportion of this association, both with (PTE $=2.66 \%, p=0.02)$ and without adjustment for lifestyles and prevalent health conditions (PTE $=$ $4.66 \%, p<0.01$; Table S3A). Associations with the "cognitive" factor were milder but still significant (Model 1: $\beta$ $(\mathrm{SE})=0.011(0.003), p<0.01$; Model $2: \beta(\mathrm{SE})=0.009$ (0.004), $p=0.01$ ), although not across all sensitivity models (Table 2). In this case, INFLA-score did not show significant explanatory proportions of association, nor did any other inflammation marker (Table S3B). Gender- and condition-stratified associations between DII and the somatic symptoms factor were concordant with those observed for the overall (PHQ9-6) symptoms score (Figure S4A- $\underline{\text { C }}$ and Table S2A and $\underline{B}$ ), while the 
A

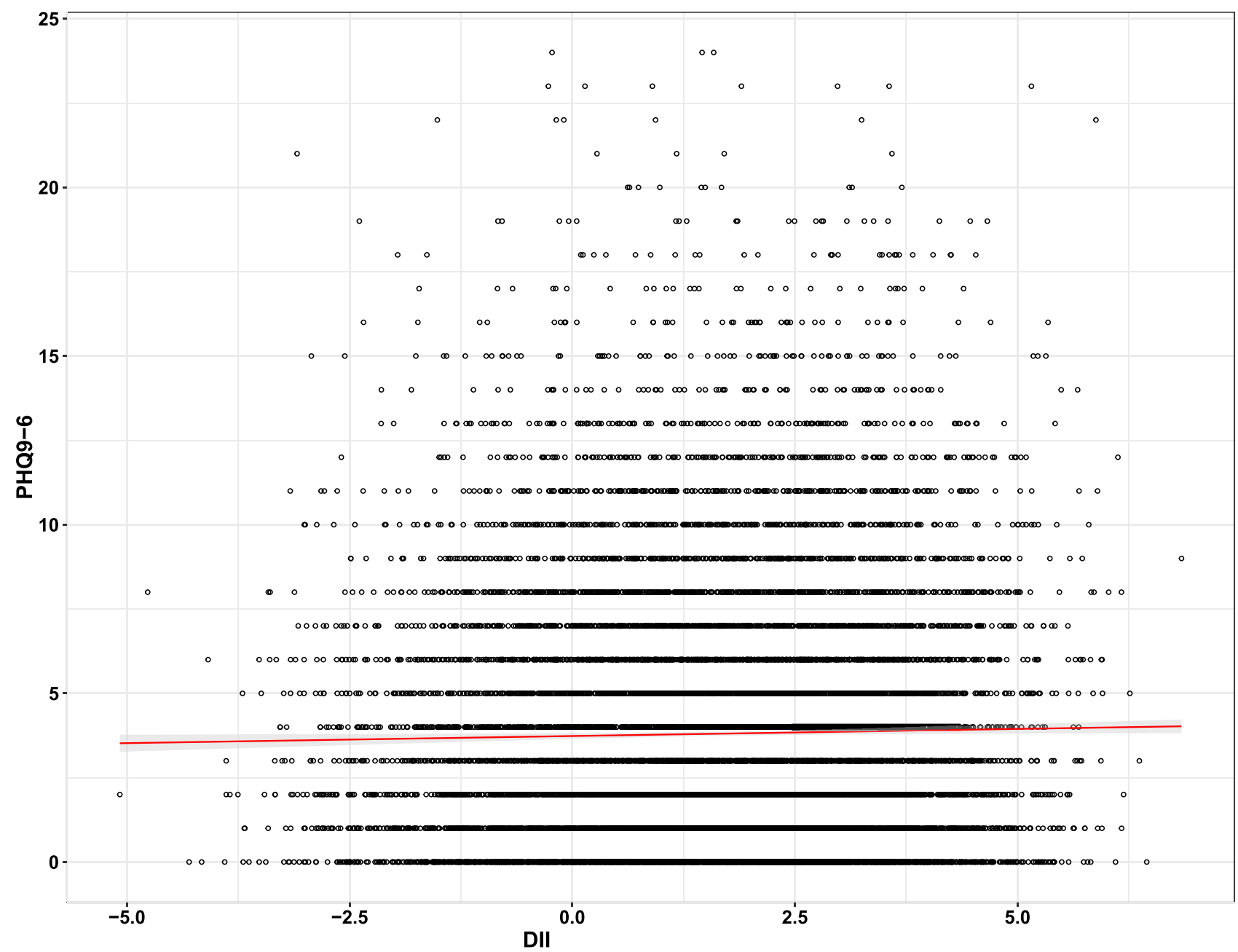

Figure I Continued.

cognitive symptoms factor showed a positive linear relation with DII within multimorbid subjects (Figure S5A-C and Table $\mathrm{S} 2 \mathrm{~A}$ and $\mathrm{B}$ ), although this was not significant due to the small sample size of the stratum (see Table S2B).

\section{Discussion}

In the present study, we analyzed the link between the DII, a quantitative measure of dietary inflammatory potential, and depressive symptoms, as well as the role of circulating inflammation biomarkers in this relationship, in a large Italian population cohort.

We identified a significant positive association between DII and depressive symptoms, in line with previous metaanalyses supporting a link of dietary inflammation with depression status, both in a cross-sectional and in a longitudinal setting. ${ }^{12,13}$ Indeed, most of the studies published so far analyzed this association comparing extremes of depressive symptoms scores distribution (eg, lower and upper tertile, quartile or quintile), or alternatively testing prediction of the incident risk of depression (reviewed $\mathrm{in}^{12,13}$ ). Here, we provide evidence of association between interindividual (continuous) variation in DII and a validated alternative version of PHQ9. Of note, a small but significant proportion of this association was explained by INFLA-score, a composite marker of circulating inflammation based on different components of the systemic inflammatory response. CRP is the most commonly used marker to evaluate circulating inflammation in humans, since it is relatively stable and easy to measure; ${ }^{38}$ increased Plt and WBC have been frequently studied as cellular inflammation indicators at the epidemiological 
B

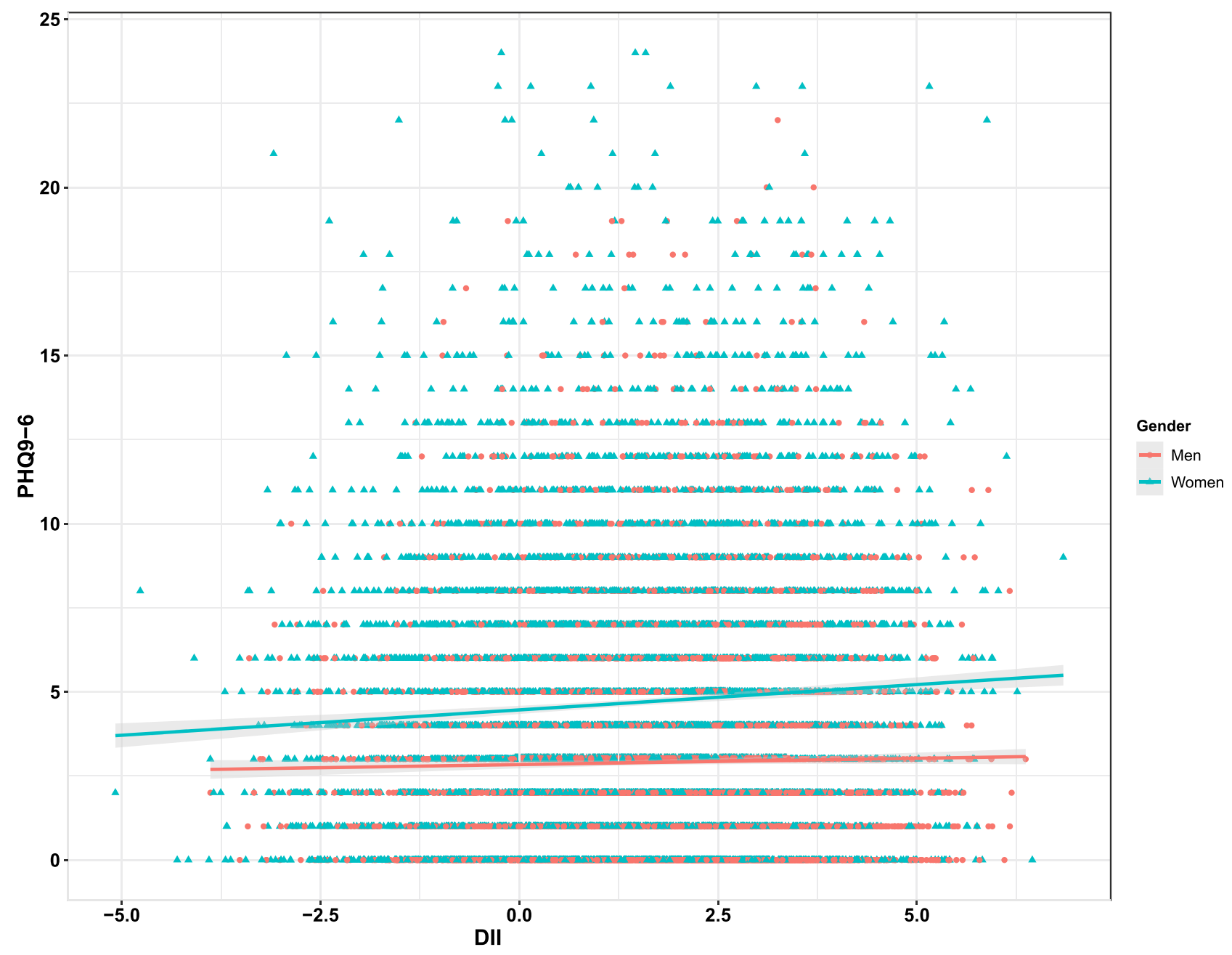

Figure I Continued.

level, ${ }^{39}$ as well as neutrophil-to-lymphocyte ratio ${ }^{40}$ which, in this study, was represented by the proxy measure GLR since neutrophils represent the majority of granulocytes $(\sim 95 \%)$ and showed a high missing rate in our cohort. ${ }^{11}$ Therefore, INFLA-score is supposed to represent a more complete marker of circulating inflammation, compared to isolated CRP or other common inflammatory cytokines like IL- 6 and TNF- $\alpha$. The proportion of association explained by INFLA-score in the link between dietary inflammation and depressive symptoms ranged between $>2 \%$ in a basic model adjusted for age and sex and $<1 \%$ in a model further adjusted for socioeconomic, lifestyle and health conditions. On the one hand, this suggests that these factors share part of the explanatory role of inflammation in the link between diet and depression, and on the other hand points towards a specific - possibly genetic - role of inflammation in this link. Indeed, a significant coheritability based on common genetic variants has been reported for CRP levels with both major depression and single depressive symptoms. ${ }^{41}$ In keeping with these findings, polygenic scores influencing higher CRP levels were positively associated with increasing altered appetite and fatigue (along with TNF- $\alpha$ ), suggesting that genetic predisposition to higher systemic inflammatory markers are primarily associated with somatic/neurovegetative symptoms of depression. ${ }^{42}$ This evidence was supported by a multi-sample network analysis in depressed and general population cohorts, and is also consistent with previous observational studies on circulating inflammation markers in population cohorts. ${ }^{7,43}$ Moreover, Mendelian randomization analyses suggested a causal effect of increased CRP on somatic symptoms (appetite alterations, tiredness/ 


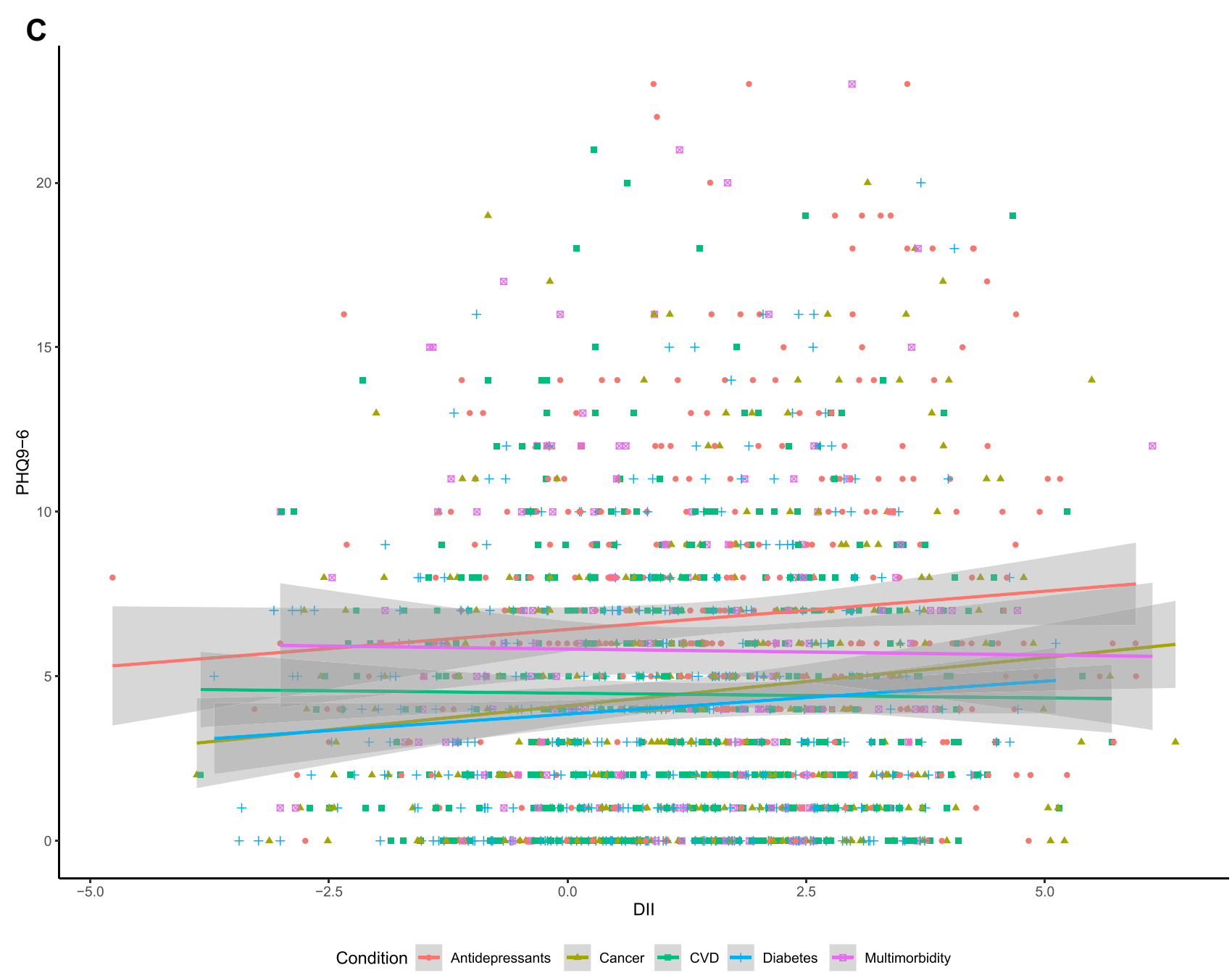

Figure I Scatter plot of overall depressive symptoms (PHQ9-6) score vs DII. Linear interpolation of data is reported for (A) the total sample $(\mathrm{N}=13,30 \mathrm{I})$, (B) separate genders and (C) prevalent health conditions or use of antidepressants, along with their $95 \%$ confidence interval (grey shade). Multimorbidity was defined as meeting two or more of the other conditions.

fatigue and psychomotor changes), and of IL-6 on cognitive symptoms (suicidality). ${ }^{41}$

In the present work, immunity-related components, prominently GLR, largely explained the association between depressive symptoms and dietary inflammation. This evidence supports for the first time a role of systemic inflammation - and in particular of the innate immunity component - in potentially mediating the hypothesized effect of nutritional patterns on depression (Figure 2), although longitudinal data will be needed to substantiate this hypothesis. Indeed, previous studies in the field identified significant associations of DII with both depression and circulating inflammatory markers like CRP, IL-6 and TNF$\alpha$, but none of them found evidence for significant direct effects of these markers in the link between depression and dietary inflammation. ${ }^{17}$ This lack of evidence has been explained through the possibility that the direct effect of diet-related inflammation is not entirely mediated by CRP or IL-6. ${ }^{18}$ Alternative pathways partly explaining the link between dietary inflammation and depressive symptoms may involve metabolic dysregulation, since it has been suggested that immune and metabolic factors may represent separate risk factors in depression, influencing partly overlapping symptoms. ${ }^{41}$ Moreover, more direct biomarkers of platelet activation - which is part of the thromboinflammatory response - and related platelet parameters may represent another potential linking pathway, also in view of recent epidemiological and genomic findings implicating them in neuropsychiatric risk, in particular depression. ${ }^{44,45}$ Another potential pathway playing such a role may be represented by gut microbiota, which has increasingly been implicated in modulating immune 
Table 3 Proportion of Total Effect (PTE) Explained by INFLA-Score ${ }^{23}$ and Its Component Biomarkers in the Association Between Dietary Inflammatory Index (DII) and Depressive Symptoms (PHQ9-6)

\begin{tabular}{|c|c|c|c|c|c|}
\hline Model & INFLA-Score & CRP & PIt & WBC & GLR \\
\hline $\mathrm{N}$ & $\begin{array}{c}2.30 \% \\
{[1.01-4.00] \%} \\
\left(<2.0 \times 10^{-16}\right)\end{array}$ & $\begin{array}{c}0.67 \% \\
{[0.00-2.00] \%} \\
(0.08)\end{array}$ & $\begin{array}{c}0.03 \% \\
{[0.00-1.00] \%(0.91)}\end{array}$ & $\begin{array}{c}0.99 \% \\
{[0.19-2.00] \%} \\
(0.01)\end{array}$ & $\begin{array}{c}1.65 \% \\
{[0.66-3.00] \%} \\
\left(<2.0 \times 10^{-16}\right)\end{array}$ \\
\hline$Y$ & $\begin{array}{c}0.90 \% \\
{[0.03-2.00] \%} \\
(0.04)\end{array}$ & $\begin{array}{c}0.10 \% \\
{[0.00-1.00] \%} \\
(0.61)\end{array}$ & $\begin{array}{c}0.05 \% \\
{[0.00-1.00] \%} \\
(0.84)\end{array}$ & $\begin{array}{c}0.00 \% \\
{[0.00-0.00](0.88)}\end{array}$ & $\begin{array}{c}1.18 \% \\
{[0.00-3.00] \%(0.01)}\end{array}$ \\
\hline
\end{tabular}

Notes: PTE $95 \%$ confidence interval $(95 \mathrm{Cl})$ and p-values are reported for INFLA-score, c-reactive protein (CRP, log scale), platelet count (PIt), white blood cell count (WBC, log scale) and granulocyte-to-lymphocyte ratio (GLR). Significant PTEs $(\mathrm{p}<0.05)$ are highlighted in bold. Model "N" was adjusted for recruiting center, sex and age, whilst model "Y" was also adjusted for education, leisure-time physical activity, smoking status, drinking habits, diabetes, cancer and cardiovascular disease (see Supplementary Methods for a description of variables). PTEs and their confidence intervals were rounded to the second decimal place, therefore some of the limits may equal zero.

response and brain functions. ${ }^{46}$ Indeed, an altered microbiota - also known as dysbiosis, a phenomenon strictly dependent on nutritional patterns - has been observed in depressed patients compared to controls (see ${ }^{47,48}$ for a review). This may contribute to the etiopathogenesis of depression through different mechanisms like i) modulating the inflammatory/immune response (eg, gut TNF- $\alpha$ secretion) and ii) regulating the metabolism of macronutrients (protein and carbohydrates) which are indirectly linked to these systems, iii) producing micronutrients influencing depressive symptoms (eg, folate, thiamine) and iv) regulating neurotransmitter pathways directly implicated in depression, like dopamine and serotonin. ${ }^{47-49}$ Experimental studies of fecal transfer in mice and of probiotics administration in humans have further supported the efficacy of microbiome modulation to regulate behavioral and cognitive traits. ${ }^{50,51}$ Overall, this evidence support the microbiome as a lens to disentangle the underlying biology of depression and as a promising target for its treatment. ${ }^{46}$

When depressive symptoms were dissected into factors representing somatic and cognitive domains, we observed significant associations in the basic and in the full model for both factors, although the somatic one showed somewhat stronger effect sizes, which survived all adjustments in sensitivity analyses. Interestingly, INFLA-score only explained a significant proportion of the association between DII and the somatic factor, suggesting that circulating inflammation may play an important role solely in

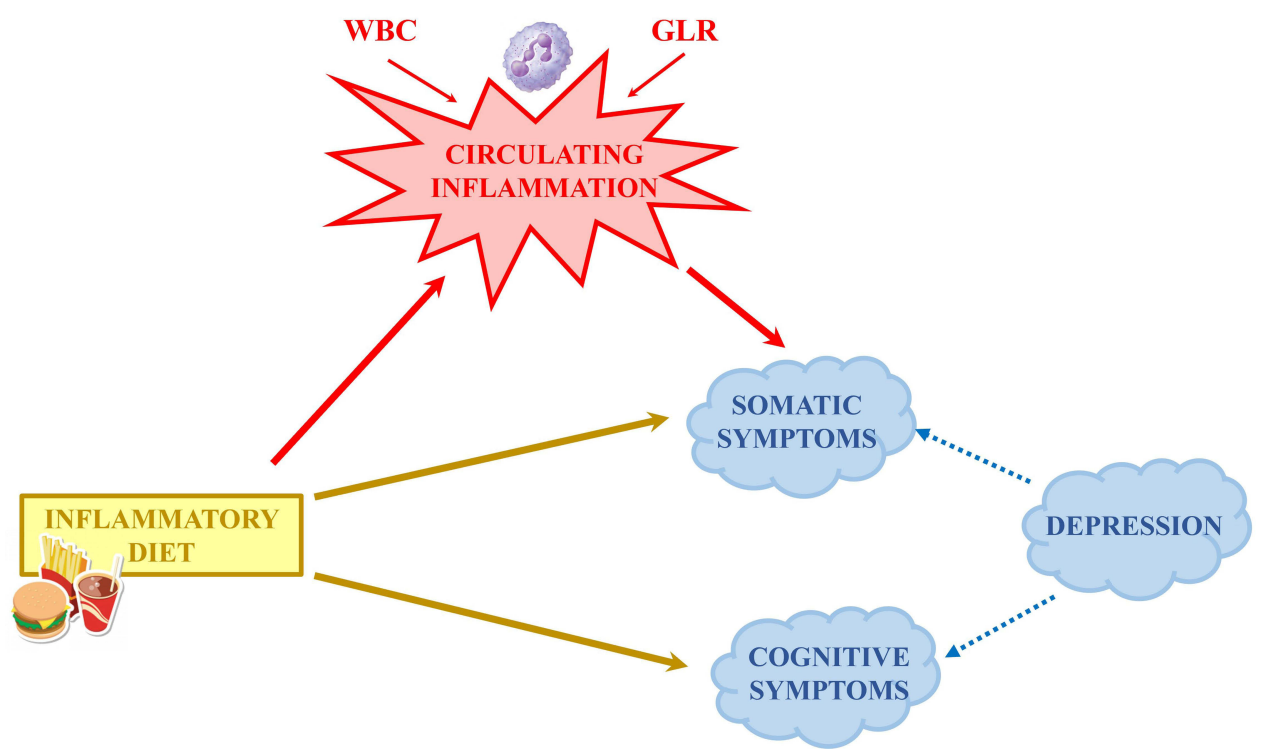

Figure 2 Hypothetical relationship between dietary inflammation and depression. In this study we report a significant association of inflammatory diet with two factors tagging somatic and cognitive symptoms of depression, in addition to a total symptom score (PHQ9-6). ${ }^{24,26}$ Circulating inflammation - in particular white blood cell count (WBC) and granulocyte-to-lymphocyte ratio (GLR) - explained part of the associations with total and somatic depressive symptoms, but not with cognitive symptoms. 
the link between pro-inflammatory diet and somatic depressive symptoms, independent of cognitive symptoms (Figure 2). To our knowledge, this is the first time such evidence has been reported. The lack of a significant explanatory effect of INFLA-score in the association between DII and the cognitive factor may be explained by the hypothesis that dietary inflammation may influence cognitive symptoms by affecting concentrations of several neurotransmitters or neuropeptides, which is only partly intertwined with circulating inflammation. In other words, accounting for circulating inflammatory markers may not be sufficient for identifying the diet-inflammation-depression relation without considering other interface mediators like neurotransmitters, neurotrophins, or neuropeptides. ${ }^{17}$ In this view, the path between inflammatory diet and depression would be diluted by the presence of different correlated pathways, with different extent of overlap with circulating inflammation, which would include multiple neurobiological factors like serotonin, norepinephrine, corticotropin-releasing and brain-derived neurotrophic factor. However, the molecular mechanisms linking an inflammatory diet with the biochemical or neurochemical mechanisms involved in the pathogenesis of depression remain largely unknown. ${ }^{17}$ Because no other study so far has examined the relationship between inflammatory diet and specific depressive domains at such a fine-grained resolution, further studies are needed to investigate this hypothesis, possibly exploiting measures of circulating neurotransmitters and validating these findings in functional in vitro and in vivo models.

Similarly, there is a need to clarify the reason why the association between dietary inflammation and depressive symptoms is stronger in women compared to men, and null in CVD and comorbid patients compared to apparently healthy subjects. While the former finding may be explained by an increased susceptibility of women to inflammation, its causes and its mood effects, ${ }^{52}$ to explain non-associations we may hypothesize that CVD and comorbid cases have reached a "plateau" inflammatory response to external stimuli, which acts as a sort of buffer against external pro-inflammatory stimuli like nutritional habits. Few is known on the complex relationship among CVD and its comorbidities, depression and inflammation, and further studies are warranted to enlighten this aspect.

\section{Strengths and Limitations}

To our knowledge, this study represents one of the largest investigations analyzing the association between the inflammatory potential of diet and depression, the largest and most comprehensive analyzing the role of circulating inflammation in this link, and the richest in terms of biomarkers tested. In particular, we analyzed a composite inflammation index conceived to capture both plasma and cellular circulating inflammation, ${ }^{23}$ which has been previously associated with both dietary inflammatory content $^{14}$ and with mortality and hospitalization risk in the Moli-sani study. ${ }^{24,53}$ This choice was further supported by the significant proportion of associations explained by immunity-related component biomarkers in the link between dietary inflammation and depressive symptoms, which once again underlines the importance of capturing different components of inflammation, not only those related to the most commonly used markers (eg CRP and IL-6). Moreover, for the first time we tested associations with factors representing two main depressive domains, namely somatic and cognitive symptoms.

Despite its strengths, some limitations apply. First, the lack of one of the items (feeling of failure) in the selfreported assessment of depressive symptoms, which however was partly counterbalanced by the moderate to strong crossitem correlations, by previous validation of the resulting deprived PHQ9-6 score in predicting depression severity $^{24,26}$ and by the factor-based approach used here to investigate relationships with specific domains. Similarly, the DII was computed over 33 out of 45 food intakes of the original version, due to the unavailability of the other foods in our nutritional assessment. This approach is quite common since it is very difficult to have complete assessment of all food intakes across different cohorts ${ }^{16,19,54-56}$ and the DII score can be calculated using $>20$ items from the desired list of food parameters. ${ }^{16}$ Second, the cross-sectional design of the study does not allow us to establish temporal components of the causal links in the association between DII and depressive symptoms scores. For the same reason, although part of these associations was explained by circulating inflammation, we cannot formally claim a mediation role and further longitudinal investigations will be needed to support it. In this perspective, reverse causality bias cannot be ruled out, since it is known that mental health may affect circulating inflammation through lifestyles ${ }^{11}$ and the link between depression and inflammation is likely bi-directional. ${ }^{57}$ However, here we focused on the known influence of dietary inflammation on depression, which has been robustly supported by several longitudinal studies. ${ }^{12,13}$ Third, any dietary self-report is associated with errors of various kinds. ${ }^{58}$ 


\section{Conclusions}

In light of the known link between atypical depression and resistance to pharmacological antidepressant treatments, ${ }^{5-7}$ if future analyses will confirm our findings in a longitudinal setting, these may suggest an alternative way to treat this depression subtype, through promoting healthy lifestyles and in particular healthy nutritional habits. In the future, this approach may be used not only for the prevention of depression onset, but also for coadjuvant treatment. This may lead to potential benefits both for national health/welfare systems and for patients, who will less likely experience side effects typically encountered in the use of psychoactive drugs. ${ }^{6}$

\section{URLs}

The R project: https://www.r-project.org/

Mediation package: https://cran.r-project.org/web/ packages/mediation/index.html

Psych package: https://cran.r-project.org/web/packages/ psych/index.html

Moli-sani study website: https://www.moli-sani.org/

\section{Data Sharing Statement}

The data underlying this article will be shared upon reasonable request to the corresponding author (alessandro.gialluisi@gmail.com). The data are stored in an institutional repository (https://repository.neuromed.it) and access is restricted by the ethical approvals and the legislation of the European Union. Moli-sani data are available to any external researcher, under approval of a research proposal and of an ancillary data exchange form by the Moli-sani Steering Committee (see https://www.moli-sani.org/?page_id=455).

\section{Acknowledgments}

We would like to thank the Moli-sani Study Investigators (see https://www.moli-sani.org/?page id=455 for a complete list) and the Associazione Cuore Sano Onlus (Campobasso, Italy) for its cultural and financial support. We thank Prof Marco Sarchiapone for inspiring and designing psychometric assessment in the Moli-sani study.

\section{Author Contributions}

AG and LI conceived and designed the present analyses. All authors contributed to data analysis, drafting or revising the article, have agreed on the journal to which the article will be submitted, gave final approval of the version to be published, and agree to be accountable for all aspects of the work.

\section{Funding}

The present work was partially supported by POR FESR 2014-2020: DD n. 45927/11/2018. SATIN: Sviluppo di Approcci Terapeutici INnovativi per patologie neoplastiche resistenti ai trattamenti; and by the Italian Ministry of Health (Ricerca Corrente 2019-2023). AG was supported by Fondazione Umberto Veronesi.Funders had no role in this study design, collection, analysis, and interpretation of data, nor in the writing and submission phase of the manuscript.

\section{Disclosure}

Dr. James R. Hébert owns controlling interest in Connecting Health Innovations LLC (CHI), a company that has licensed the right to his invention of the dietary inflammatory index $\left(\mathrm{DII}^{\mathbb{B}}\right.$ ) from the University of South Carolina in order to develop computer and smart phone applications for patient counseling and dietary intervention in clinical settings. Dr Nitin Shivappa is an employee of CHI. All other authors declare no competing financial interests.

\section{References}

1. World Health Organization. Depression and other common mental disorders: global health estimates; 2017.

2. Hasin DS, Sarvet AL, Meyers JL, et al. Epidemiology of adult DSM-5 major depressive disorder and its specifiers in the United States. JAMA Psychiatry. 2018;75:336-346.

3. Werner F-M, Coveñas R. Classical neurotransmitters and neuropeptides involved in major depression in a multi-neurotransmitter system: a focus on antidepressant drugs. Curr Med Chem. 2013;20 (38):4853-4858. doi:10.2174/09298673113206660280.

4. Voineskos D, Daskalakis ZJ, Blumberger DM. Management of treatment-resistant depression: challenges and strategies. Neuropsychiatr Dis Treat. 2020;16:221-234. doi:10.2147/NDT. S198774

5. Łojko D, Rybakowski JK. Atypical depression: current perspectives. Neuropsychiatr Dis Treat. 2017;13:2447-2456. doi:10.2147/NDT. S147317

6. Sen ZD, Danyeli LV, Woelfer M, et al. Linking atypical depression and insulin resistance-related disorders via low-grade chronic inflammation: integrating the phenotypic, molecular and neuroanatomical dimensions. Brain Behav Immun. 2021. doi:10.1016/j.bbi.2020. 12.020

7. Gialluisi A, Di Castelnuovo A, Bracone F, et al. Associations between systemic inflammation and somatic depressive symptoms: findings from the Moli-sani study. Depress Anxiety. 2020;37 (9):935-943. doi:10.1002/da.23070

8. Chamberlain SR, Cavanagh J, De BP, et al. Treatment-resistant depression and peripheral C-reactive protein. $\mathrm{Br} J$ Psychiatry. 2019;214(1):11-19. doi:10.1192/bjp.2018.66

9. Uher R, Tansey KE, Dew T, et al. An inflammatory biomarker as a differential predictor of outcome of depression treatment with escitalopram and nortriptyline. Am $J$ Psychiatry. 2014;171 (12):1278-1286. doi:10.1176/appi.ajp.2014.14010094

10. Miller AH, Raison CL. The role of inflammation in depression: from evolutionary imperative to modern treatment target. Nat Rev Immunol. 2016;16(1):22-34. doi:10.1038/nri.2015.5. 
11. Gialluisi A, Bonaccio M, Di Castelnuovo A, et al. Lifestyle and biological factors influence the relationship between mental health and low-grade inflammation. Brain Behav Immun. 2020;85:4-13. doi:10.1016/j.bbi.2019.04.041

12. Lassale C, Batty GD, Baghdadli A, et al. Healthy dietary indices and risk of depressive outcomes: a systematic review and meta-analysis of observational studies. Mol Psychiatry. 2018;1. Available from: http://www.nature.com/articles/s41380-018-0237-8.

13. Tolkien K, Bradburn S, Murgatroyd C. An anti-inflammatory diet as a potential intervention for depressive disorders: a systematic review and meta-analysis. Clin Nutr. 2019;38(5):2045-2052. doi:10.1016/j. clnu.2018.11.007

14. Shivappa N, Bonaccio M, Hebert JR, et al. Association of proinflammatory diet with low-grade inflammation: results from the Moli-sani study. Nutrition. 2018;54:182-188. doi:10.1016/j.nut.2018.04.004

15. Phillips CM, Shivappa N, Hébert JR, Perry IJ. Dietary inflammatory index and biomarkers of lipoprotein metabolism, inflammation and glucose homeostasis in adults. Nutrients. 2018;10(8):1-13. doi:10. 3390/nu10081033

16. Corley J, Shivappa N, Hébert JR, Starr JM, Deary IJ. Associations between dietary inflammatory index scores and inflammatory biomarkers among older adults in the Lothian birth cohort 1936 study. J Nutr Health Aging. 2019;23(7):628-636. doi:10.1007/s12603-019-1221-y

17. Kheirouri S, Alizadeh M. Dietary inflammatory potential and the risk of incident depression in adults: a systematic review. Adv Nutr. 2019;10(1):9-18. doi:10.1093/advances/nmy100.

18. Akbaraly T, Kerlau C, Wyart M, et al. Dietary inflammatory index and recurrence of depressive symptoms: results from the Whitehall II study. Clin Psychol Sci. 2017;4:1125-1134.

19. Phillips CM, Shivappa N, Hébert JR, Perry IJ. Dietary inflammatory index and mental health: a cross-sectional analysis of the relationship with depressive symptoms, anxiety and well-being in adults. Clin Nutr. 2018;37(5):1485-1491. doi:10.1016/j.clnu.2017.08.029

20. Jorgensen D, White GE, Sekikawa A, Gianaros P. Higher dietary inflammation is associated with increased odds of depression independent of Framingham risk score in the national health and nutrition examination survey. Nutr Res. 2018;54:23-32. doi:10.1016/j.nutres. 2018.03.004

21. Vermeulen E, Brouwer IA, Stronks K, et al. Inflammatory dietary patterns and depressive symptoms in Italian older adults. Brain Behav Immun. 2018;67:290-298. doi:10.1016/j.bbi.2017.09.005

22. Di Castelnuovo A, Costanzo S, Persichillo M, et al. Distribution of short and lifetime risks for cardiovascular disease in Italians. Eur J Prev Cardiol. 2012;19(4):723-730. doi:10.1177/1741826711410820

23. Pounis G, Bonaccio M, Di Castelnuovo A, et al. Polyphenol intake is associated with low-grade inflammation, using a novel data analysis from the Moli-sani study. Thromb Haemost. 2016;115(2):344-352. doi:10.1160/th15-06-0487

24. Gialluisi A, Costanzo S, Di CA, et al. Combined influence of depression severity and low-grade inflammation on incident hospitalization and mortality risk in Italian adults. J Affect Disord. 2020;27 9:173-182 Available from https://linkinghub.elsevier.com/retrieve/ pii/S0165032720328482.

25. Kroenke K, Spitzer RL, Williams JBW. The PHQ-9: validity of a brief depression severity measure. J Gen Intern Med. 2001;16 (9):606-613. doi:10.1046/j.1525-1497.2001.016009606.x

26. Gialluisi A. Corrigendum to "Combined influence of depression severity and low-grade inflammation on incident hospitalization and mortality risk in Italian adults" (Journal of Affective Disorders, Volume 279, 15 January 2021, Pages 173-182). J Affect Disord. 2021;292:790. doi:10.1016/j.jad.2021.06.031

27. R Core Team. R: a language and environment for statistical computing; 2020. Available from: https://www.r-project.org/. Accessed September $15,2021$.
28. Tsoupras A, Lordan R, Zabetakis I. Inflammation, not cholesterol, is a cause of chronic disease. Nutrients. 2018;10(5):604. doi:10.3390/ nu10050604

29. Sartorius N. Depression and diabetes. Dialogues Clin Neurosci. 2018;20(1):47.

30. Elderon L, Whooley MA. Depression and cardiovascular disease. Prog Cardiovasc Dis. 2013;55(6):511-523. doi:10.1016/j.pcad.20 13.03.010

31. Friedland CJ. Head and neck cancer: identifying depression as a comorbidity among patients. Clin J Oncol Nurs. 2019;23 (1):99-102.

32. Rice SM, Oliffe JL, Kelly MT, et al. Depression and prostate cancer: examining comorbidity and male-specific symptoms. Am J Mens Health. 2018;12(6):1864-1872. doi:10.1177/1557988318784395

33. Connor KM, Davidson JRT. Development of a new resilience scale: the Connor-Davidson Resilience Scale (CD-RISC). Depress Anxiety. 2003;18(2):76-82. doi:10.1002/da.10113

34. Trichopoulou A, Costacou T, Bamia C, Trichopoulos D. Adherence to a Mediterranean diet and survival in a Greek population. $N$ Engl J Med. 2003;348(26):2599-2608. doi:10.1056/NEJMoa025039.

35. Woolcott OO, Bergman RN. Relative fat mass (RFM) as a new estimator of whole-body fat percentage - A cross-sectional study in American adult individuals. Sci Rep. 2018;8(1):1-11. doi:10.1038/ s41598-018-29362-1

36. Bonaccio M, Di Castelnuovo A, Costanzo S, et al. Mediterraneantype diet is associated with higher psychological resilience in a general adult population: findings from the Moli-sani study. Eur J Clin Nutr. 2018;72(1):154-160. doi:10.1038/ejen.2017.150

37. D'Esposito V, Ambrosio MR, Liguoro D, et al. In severe obesity, subcutaneous adipose tissue cell-derived cytokines are early markers of impaired glucose tolerance and are modulated by quercetin. Int J Obes. 2021;45(8):1811-1820. doi:10.1038/s41366-021-00850-1

38. Danesh J, Wheeler JG, Hirschfield GM, et al. C-reactive protein and other circulating markers of inflammation in the prediction of coronary heart disease. $N$ Engl J Med. 2004;350(14):1387-1397. doi:10.1056/NEJMoa032804

39. Bonaccio M, Di Castelnuovo A, De Curtis A, et al. Adherence to the Mediterranean diet is associated with lower platelet and leukocyte counts: results from the Moli-sani study. Blood. 2014;123 (19):3037LP-3044LP. doi:10.1182/blood-2013-12-541672

40. Guthrie GJK, Charles KA, Roxburgh CSD, Horgan PG, McMillan DC, Clarke SJ. The systemic inflammation-based neutrophil-lymphocyte ratio: experience in patients with cancer. Crit Rev Oncol Hematol. 2013;88(1):218-230. doi:10.1016/j.critrevonc.2013.03.010.

41. Kappelmann N, Arloth J, Georgakis MK, et al. Dissecting the association between inflammation, metabolic dysregulation, and specific depressive symptoms: a genetic correlation and 2-sample Mendelian randomization study. JAMA Psychiatry. 2021;78(2):161-170. doi:10.1001/jamapsychiatry.2020.3436

42. Kappelmann N, Czamara D, Rost N, et al. Polygenic risk for immuno-metabolic markers and specific depressive symptoms: a multi-sample network analysis study. Brain Behav Immun. 2021;95:256-268. doi:10.1016/j.bbi.2021.03.024

43. Milaneschi Y, Kappelmann N, Ye Z, et al. Association of inflammation with depression and anxiety: evidence for symptom-specificity and potential causality from UK Biobank and NESDA cohorts. Mol Psychiatry. 2021; 1-10. doi:10.1038/s41380-021-01188-w.

44. Izzi B, Tirozzi A, Cerletti C, et al. Beyond haemostasis and thrombosis: platelets in depression and its co-morbidities. Int $\mathrm{J} \mathrm{Mol} \mathrm{Sci}$. 2020;21(22)1-32.

45. Gialluisi A, Izzi B, Di Castelnuovo A, et al. Revisiting the link between platelets and depression through genetic epidemiology: new insights from platelet distribution width. Haematologica. 2019;105(5):e246. 
46. Beurel E, Toups M, Nemeroff CB. The bidirectional relationship of depression and inflammation: double trouble. Neuron. 2020;107:234-256.

47. Cheung SG, Goldenthal AR, Uhlemann A-C, Mann JJ, Miller JM, Sublette ME. Systematic review of gut microbiota and major depression. Front Psychiatry. 2019;10:34. doi:10.3389/fpsyt.2019.00034

48. Rogers GB, Keating DJ, Young RL, Wong M-L, Licinio J, Wesselingh S. From gut dysbiosis to altered brain function and mental illness: mechanisms and pathways. Mol Psychiatry. 2016;21:738-748.

49. Valles-Colomer M, Falony G, Darzi Y, et al. The neuroactive potential of the human gut microbiota in quality of life and depression. Nat Microbiol. 2019;4(4):623-632. doi:10.1038/s41564-018-0337-X

50. Cruz-Pereira JS, Rea K, Nolan YM, O’Leary OF, Dinan TG, Cryan JF. Depression's unholy trinity: dysregulated stress, immunity, and the microbiome. Annu Rev. 2020; 71:49-78. doi:10.1146/ annurev-psych-122216-011613.

51. Sanada K, Nakajima S, Kurokawa S, et al. Gut microbiota and major depressive disorder: a systematic review and meta-analysis. J Affect Disord. 2020;266:1-13. doi:10.1016/j.jad.2020.01.102

52. Derry HM, Padin AC, Kuo JL, Hughes S, Kiecolt-Glaser JK. Sex differences in depression: does inflammation play a role? Curr Psychiatry Rep. 2015;17(10):78. doi:10.1007/s11920-015-0618-5.

53. Bonaccio M, Di Castelnuovo A, Pounis G, et al. A score of low-grade inflammation and risk of mortality: prospective findings from the moli-sani study. Haematologica. 2016;101(11):1434-1441. doi:10.33 24/haematol.2016.144055
54. Shivappa N, Steck SE, Hurley TG, et al. A population-based dietary inflammatory index predicts levels of C-reactive protein in the Seasonal Variation of Blood Cholesterol Study (SEASONS). Public Health Nutr. 2014;17(8):1825-1833. doi:10.1017/S136898001300 2565

55. Salari-Moghaddam A, Keshteli AH, Afshar H, Esmaillzadeh A, Adibi P. Association between dietary inflammatory index and psychological profile in adults. Clin Nutr. 2019;38(5):2360-2368. doi:10.1016/j.clnu.2018.10.015

56. Mickle AT, Brenner DR, Beattie T, Williamson T, Courneya KS, Friedenreich CM. The dietary inflammatory index ${ }^{\circledR}$ and alternative healthy eating index 2010 in relation to leucocyte telomere length in postmenopausal women: a cross-sectional study. J Nutr Sci. 2019;8: e35. doi:10.1017/jns.2019.32

57. Matthews KA, Schott LL, Bromberger JT, Cyranowski JM, EversonRose SA, Sowers M. Are there bi-directional associations between depressive symptoms and C-reactive protein in mid-life women? Brain Behav Immun. 2010;24(1):96-101. doi:10.1016/j.bbi.2009.08. 005 .

58. Hebert JR, Clemow L, Pbert L, Ockene IS, Ockene JK. Social desirability bias in dietary self-report may compromise the validity of dietary intake measures. Int $J$ Epidemiol. 1995;24(2):389-398. doi:10.1093/ije/24.2.389
Journal of Inflammation Research

\section{Publish your work in this journal}

The Journal of Inflammation Research is an international, peerreviewed open-access journal that welcomes laboratory and clinical findings on the molecular basis, cell biology and pharmacology of inflammation including original research, reviews, symposium reports, hypothesis formation and commentaries on: acute/chronic inflammation; mediators of inflammation; cellular processes; molecular

\section{Dovepress}

mechanisms; pharmacology and novel anti-inflammatory drugs; clinical conditions involving inflammation. The manuscript management system is completely online and includes a very quick and fair peerreview system. Visit http://www.dovepress.com/testimonials.php to read real quotes from published authors. 